\title{
Plasma Endotoxin Levels Are Not Increased in Schistosoma mansoni-Infected Women without Signs or Symptoms of Hepatosplenic Disease
}

\author{
Katherine M. Klemperer, ${ }^{1,2}$ Mary Juliet Reust, ${ }^{1}$ Myung Hee Lee, ${ }^{1}$ Paul L. A. M. Corstjens, ${ }^{3}$ Govert J. van Dam, ${ }^{4}$ \\ Humphrey D. Mazigo, ${ }^{5}$ Kathryn M. Dupnik, ${ }^{1}$ and Jennifer A. Downs ${ }^{1,6 *}$ \\ ${ }^{1}$ Department of Medicine, Weill Cornell Medicine, New York, New York; ${ }^{2}$ St John's College, Cambridge University, Cambridge, United Kingdom; \\ ${ }^{3}$ Department of Cell and Chemical Biology, Leiden University Medical Center, Leiden, The Netherlands; ${ }^{4}$ Department of Parasitology, Leiden \\ University Medical Center, Leiden, The Netherlands; ${ }^{5}$ Department of Parasitology, Catholic University of Health and Allied Sciences, Mwanza, \\ Tanzania; ${ }^{6}$ Department of Medicine, Bugando Medical Center, Mwanza, Tanzania
}

\begin{abstract}
Elevated circulating endotoxin levels in the plasma of patients with advanced hepatosplenic schistosomiasis caused by Schistosoma mansoni have been reported, possibly caused by parasite egg-induced intestinal mucosal breaches facilitating bacterial access to the bloodstream. Neither endotoxin levels in people with S. mansoni but without hepatosplenic disease nor the impact of treatment on endotoxin levels have been described. We used a methodically optimized Limulus amebocyte lysate assay to measure plasma endotoxin in community-dwelling women from an S. mansoni-endemic area without clinical hepatosplenic disease. We found no difference in baseline mean plasma endotoxin levels between those with $(n=22)$ and without $(n=31)$ infection (1.001 versus $0.949 \mathrm{EU} / \mathrm{mL}, P=0.61)$. Endotoxin levels did not change in schistosome-infected women after successful treatment (1.001 versus $1.093 \mathrm{EU} / \mathrm{mL}, P=0.45$ ) and were not correlated with circulating anodic antigen or stool egg burden. Our findings do not support the hypothesis that translocating eggs in S. mansoni infection introduce bacterial sources of endotoxin to the circulation.
\end{abstract}

Schistosomiasis impacts more than 200 million people worldwide. Morbidity in Schistosoma mansoni infection is largely attributable to migrating eggs causing inflammation in gastrointestinal and hepatic tissues. In patients with advanced hepatosplenic schistosomiasis, elevated endotoxin levels have been reported and are hypothesized to be due to egg-induced breaches in the intestinal mucosa, which facilitate translocation of lipopolysaccharide-positive gastrointestinal bacteria from the lumen of the gut into the mucosa and surrounding tissues. ${ }^{1,2}$ Comparisons of endotoxin levels between individuals are hampered by heterogeneity in endotoxin molecules and plasma interference by other substances. ${ }^{3,4}$ Given reported associations of endotoxemia with morbidity, ${ }^{4,5}$ understanding its effects in schistosome infection is critical.

Because comparing individuals' endotoxin levels longitudinally may lessen issues of heterogeneity, we undertook the first prospective study of endotoxin levels pre- and postpraziquantel treatment for $S$. mansoni infections. We enrolled patients who had detectable parasite eggs in stool without clinical evidence of hepatosplenic involvement to determine whether endotoxemia could be associated with $S$. mansoni infection.

Samples were collected every 3 months from 54 women living in a S. mansoni-endemic region of northwest Tanzania over a 12-month period (June 2017-June 2018). Women provided written informed consent for participation in a longitudinal study of schistosomiasis in the gastrointestinal and genital tracts. Ethical approvals were obtained from the Joint Research Ethics Committee at Bugando Medical Centre and the Catholic University of Health and Allied Sciences and the National Institute for Medical Research (both in Tanzania) and from Weill Cornell Medicine. We analyzed data from all women who attended both the first two study visits. Women

${ }^{*}$ Address correspondence to Jennifer A. Downs, Weill Cornell Medicine Center for Global Health, 402 E 67th St., 2nd floor, New York, NY 10065. E-mail: jna2002@med.cornell.edu received free treatment for all helminth and genital tract infections diagnosed. None had clinical evidence of ascites or edema.

Schistosome infection was diagnosed, as previously described, using serum circulating anodic antigen (CAA), with a positivity threshold of $30 \mathrm{pg} / \mathrm{mL}^{6}$, and urine and stool microscopy. Each participant provided one stool sample from which five Kato-Katz slides were prepared, ${ }^{7}$ and one urine sample from which $10 \mathrm{~mL}$ was filtered for microscopic examination by a trained parasitologist. Those enrolled in this study were positive for $S$. mansoni infection both by stool eggs and by elevated serum CAA levels.

Blood was drawn into potassium ethylenediaminetetraacetic acid (EDTA) vacutainer tubes (Becton Dickinson, Franklin Lakes, $\mathrm{NJ}$ ). Plasma was separated and stored at $-20^{\circ} \mathrm{C}$ in Mwanza, shipped on dry ice to Weill Cornell in New York in July 2018, and thereafter stored at $-80^{\circ} \mathrm{C}$. The samples were tested between August and November 2018. Endotoxin levels were, thus, assayed between 6 weeks and 13 months after phlebotomy.

Endotoxin concentration was quantified via turbidimetric kinetic Pyrotell-T Limulus amebocyte lysate (LAL) assay (Associates of Cape Cod, East Falmouth, MA), as per the manufacturer's instructions. The LAL assay was reconstituted with Glucashield buffer to minimize interference from glucans. Each set of assays included internal positive controls for each specimen, from which $50-200 \%$ of a known spike quantity must be recovered to be considered valid, ${ }^{8}$ and a standard curve was constructed from ACC control standard endotoxin (lot \#160).

To ensure that our sample collection method minimized interference, ${ }^{9-11}$ we compared endotoxin concentrations in eight people with blood collected simultaneously in EDTA or heparin tubes (each run in triplicate). All EDTA tubes had coefficients of variation (CVs) within $20 \%$, whereas only $75 \%$ of heparin tubes did (EDTA/heparin correlation $=0.4322, P=$ $0.196)$. Therefore, we analyzed endotoxin levels in blood collected in EDTA.

Samples were originally diluted in LAL water to concentrations between $1: 10$ and $1: 5,000$ to determine which 
dilution best overcame interference. However, no concentration consistently overcame interference. To determine which heat treatment best overcame interference, samples were diluted to the concentration of $1: 2$ and placed in either boiling water for 2 minutes ${ }^{11}$ or into a $60^{\circ} \mathrm{C}$ heat block for 30 minutes. ${ }^{12}$ After cooling, 1:4 and 1:40 dilutions were created from the 1:2 dilution, and endotoxin levels were measured in all three dilutions. Results from all three dilutions showed good spike recovery and consistency between endotoxin levels measured at different dilutions, indicating that both treatments sufficiently overcame inhibition. Because boiling produced visibly coagulated samples, $60^{\circ} \mathrm{C}$ treatment was selected for use. Sample dilution to $1: 100$ followed by $60^{\circ} \mathrm{C}$ heat treatment yielded the best spike recovery percentages, had an onset time within the standard curve, and had low CV values.

We included two negative controls: 1) LAL water and 2) LAL water with Pyrotell-T. Test plates were considered valid if 1) the endotoxin readings for the negative controls were lower than those for the lowest standard and 2) the absolute value of the correlation coefficient for the standard curve was $\geq 0.980$. $^{8}$ In addition, we required the CV between triplicate samples to be $<20 \%$. $^{13}$ Samples not meeting these criteria were rerun. Using a 1:100 dilution of sample heat-treated at $60^{\circ} \mathrm{C}$ for 30 minutes, $92 \%$ of samples passed quality control. In the remaining samples $(n=5)$, we were unable to produce valid spike recovery rates after two attempts. These samples were excluded from further analysis.

We compared proportions using chi-squared tests and continuous variables by Wilcoxon rank-sum test. We performed linear regression to determine associations between endotoxin levels and intensity of schistosome infection (quantified in separate regressions by log CAA and absolute egg counts). We used cluster-robust standard errors to account for within-patient correlation across time points. Longitudinal endotoxin levels were analyzed using linear mixed models with time and schistosome infection fixed effects and patient-specific random effects to account for within-patient correlation across time points.

Women with S. mansoni infection were not significantly different from those without, except that they less frequently reported having ever received anti-schistosome treatment (Table 1). Baseline endotoxin levels were comparable
(1.001 versus $0.949 \mathrm{EU} / \mathrm{mL}, P=0.61$ ). The endotoxin level did not change in schistosome-infected women who received praziquantel and were schistosome-negative at month 3 (1.001 versus $1.093 \mathrm{EU} / \mathrm{mL}, P=0.451$, Figure 1A). Linear regression analyses indicated that neither serum CAA nor stool egg burden was associated with the endotoxin level ( $\log (\mathrm{CAA})$ coefficient $=-0.004, P=0.74$; eggs coefficient $=-0.0005, P=0.69$, respectively, Figure $1 \mathrm{~B}$ and $\mathrm{C})$. Endotoxin levels also did not differ by report of recent food insecurity (1.028 versus $0.927 \mathrm{EU} / \mathrm{mL}$, $P=0.32)$.

However, the time of sample collection did have an impact: overall endotoxin levels were higher at month 3 than baseline (Figure 1D, mean endotoxin difference $=0.17, P=0.0037$ ). By contrast, month 6 and 9 endotoxin levels were unchanged from baseline (month 6 difference $=-0.044, P=0.46$ and month 9 difference $=-0.037, P=0.56$ ).

Therefore, after rigorously optimizing the $L A L$ assay, we found no relationship between $S$. mansoni infection, infection intensity, or anti-schistosome treatment on blood endotoxin levels in our study population. This suggests that although we presume that eggs are passing through the intestinal mucosa in these S. mansoni-infected women, this movement does not affect endotoxin levels.

Our implementation of a carefully optimized protocol for endotoxin testing strengthens confidence in the validity of our findings. ${ }^{4}$ Differing assay optimizations by different investigators may explain varied associations of endotoxin levels with S. mansoni results ${ }^{14}$ particularly because testing conditions are not always described in detail. Alternatively, patients in previous studies had hepatosplenic schistosomiasis, and endotoxin elevations may be related to hepatic disease. Putative mechanisms for elevations related to hepatic disease could be impaired hepatic clearance of endotoxin or increased translocation of intestinal bacteria to the systemic circulation due to increased gut permeability in the setting of portal hypertension and ascites. ${ }^{4,13}$

Malnutrition can also affect gut permeability. ${ }^{15}$ We found no association between food insecurity and endotoxin possibly because we only inquired about food insecurity in the last month. Many participants may have suffered from malnutrition at differing points in their lifetime and seasons of the year. ${ }^{16}$ This could explain the 3 -fold higher median

TABLE 1

Baseline clinical and demographic characteristics of 53 women in a Schistosoma mansoni-endemic region of Tanzania

\begin{tabular}{|c|c|c|c|}
\hline & Schistosoma-infected $(N=22)$ & Schistosoma-noninfected $(N=31)$ & $P$-value of difference \\
\hline Age (years), median [IQR] & $31[23-39]^{*}$ & $32[23-42]$ & 0.65 \\
\hline Positive for hookworms, $n(\%)$ & $5(22.7)$ & $3(9.7)$ & 0.25 \\
\hline Number of years in school, median [IQR] & $7[4-7]^{*}$ & $7[5-7]$ & 0.63 \\
\hline $\begin{array}{l}\text { Previously treated for schistosomiasis, } \\
n(\%)\end{array}$ & $3(13.6)^{\star}$ & $13(41.9)$ & 0.036 \\
\hline Earns own money, $n(\%)$ & $11(50)^{\star}$ & $10(33.3)$ & 0.23 \\
\hline $\begin{array}{l}\text { Skipped lunch/dinner within last month } \\
\text { because of lack of food, } n(\%)\end{array}$ & $14(63.6)^{\star}$ & $13(43.3)$ & 0.15 \\
\hline Positive for HIV & 0 & 0 & $-†$ \\
\hline $\begin{array}{l}\text { Schistosome circulating anodic antigen } \\
\text { (pg/mL), median [IQR] }\end{array}$ & $3,333.1[635.7-50,000]$ & $1[0-9.2]$ & $-\dagger$ \\
\hline $\begin{array}{l}\text { Schistosoma haematobium eggs seen in } \\
\text { urine }\end{array}$ & $0^{\star}$ & 0 & $-\dagger$ \\
\hline S. mansoni eggs/g in stool, median [IQR] & $33.3[16.8-64.8]$ & 0 & $-\dagger$ \\
\hline
\end{tabular}


A

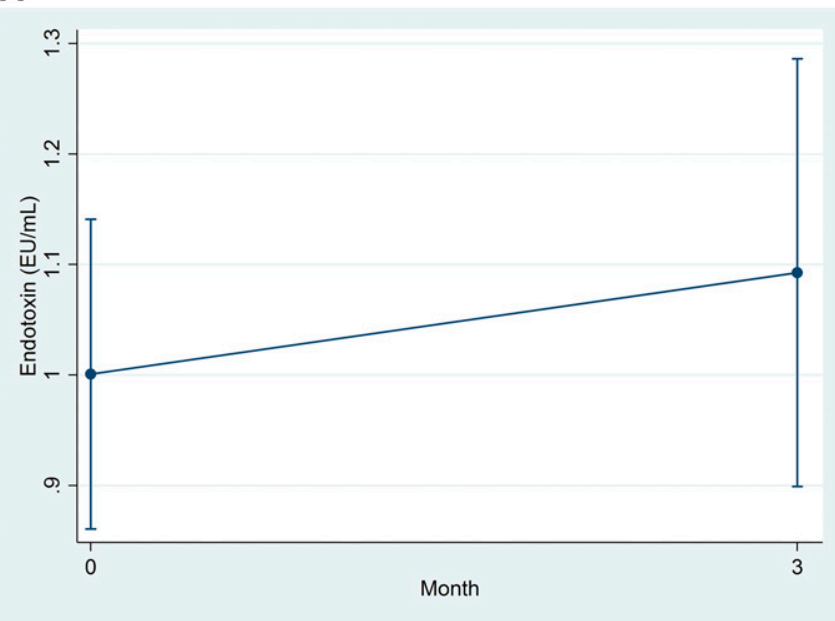

C

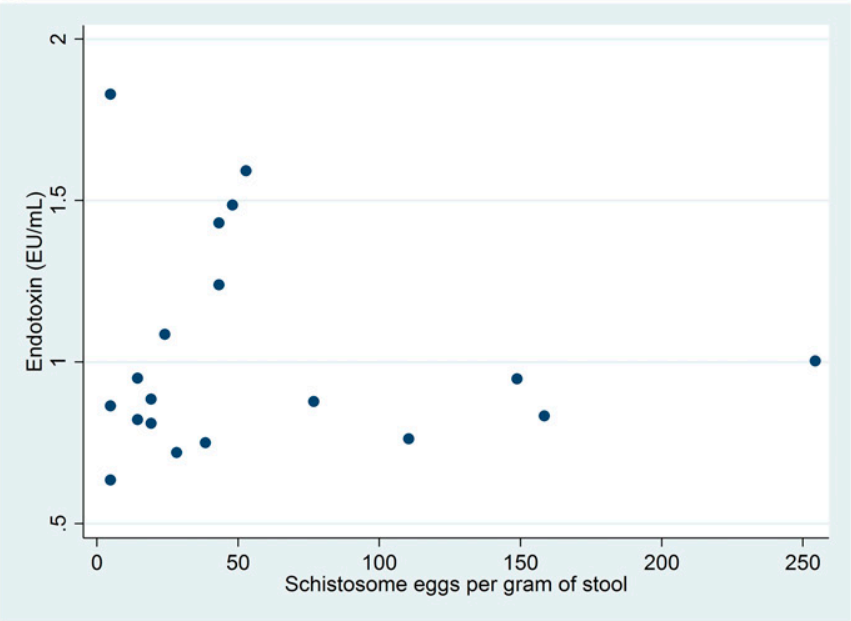

B

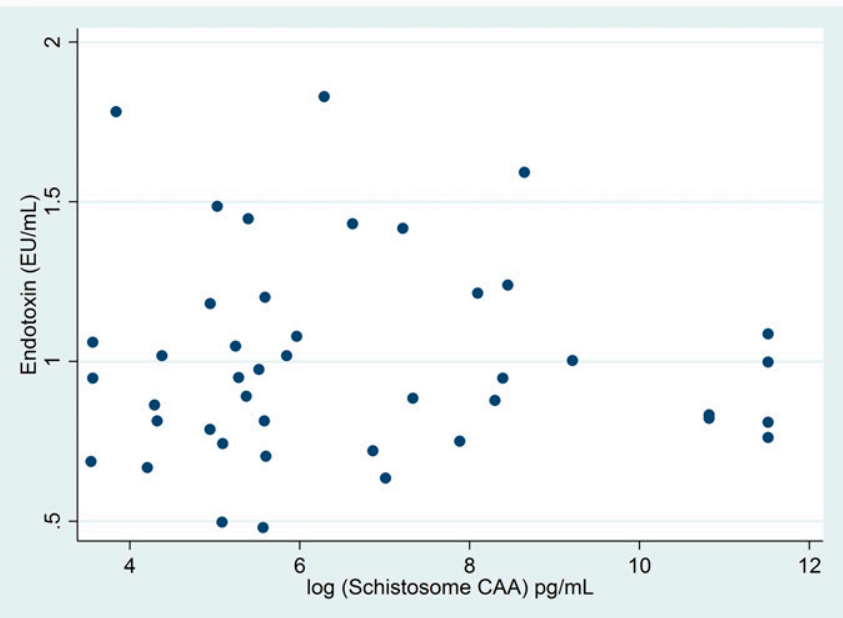

D

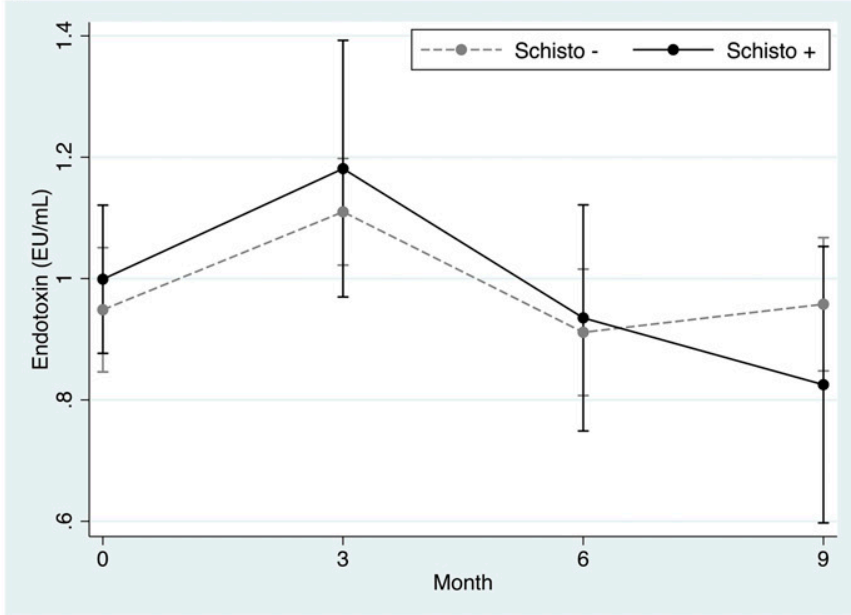

FIGURE 1. Endotoxin levels do not change after treatment, do not correlate with schistosome circulating anodic antigen (CAA) or stool eggs, and do not differ significantly by infection status over time. (A) For schistosome-infected women who became negative at month 3 after treatment: comparison of endotoxin levels between baseline and month 3. (B) For all positive samples: plot of the data used for regression of endotoxin levels on log CAA; a linear regression model with cluster-robust standard errors was used to account for within-patient correlation across time points. Each dot represents a different set of measurements. (C) For participants positive for eggs: plot of the data used for regression of endotoxin levels on stool eggs/g (eggs were only found at baseline). (D) For all samples: comparison over time of plasma endotoxin levels between those with and without schistosome infections; schistosome infection status was reassigned at each time point. A linear mixed model, with time and schistosome infection fixed effects and patient-specific random effects, was used to account for within-patient correlation across time.

endotoxin level $(0.989 \mathrm{EU} / \mathrm{mL})$ in this study compared with the median endotoxin level of $0.29 \mathrm{EU} / \mathrm{mL}$ derived from 11 studies of healthy people who used similar LAL optimizations. ${ }^{4}$

Endotoxin levels were higher in both groups at month 3 visits, which all occurred at the beginning of the rainy season, between September and December. As malaria and diarrheal disease increase during wet seasons, ${ }^{17,18}$ it is possible that local disease outbreaks during this period could have increased the overall median endotoxin levels ${ }^{19,20}$ compared with levels observed at other time points. We did not have data to assess contributions of these and other pathogens to endotoxemia.

In conclusion, S. mansoni infection in women without evidence of hepatosplenic involvement is not associated with elevated plasma endotoxin levels. The absence of endotoxemia, despite egg-induced mucosal breaches, may be attributable to complex immune mechanisms at the intestinal surface and merits further study.
Received November 27, 2019. Accepted for publication January 23 , 2020.

Published online March 2, 2020.

Acknowledgment: We thank the women for their enthusiastic participation in this project.

Financial support: This independent research was supported by the Doris Duke Charitable Foundation (Grant \#2017067), the Gilead Sciences Research Scholars Program in HIV from Gilead Sciences, and NIH K23 Al 110238.

Authors' addresses: Katherine M Klemperer, St John's College, Cambridge University, Cambridge, United Kingdom, E-mail: katherineklemperer@ googlemail.com. Mary Juliet Reust, Myung Hee Lee, and Kathryn M. Dupnik, Department of Medicine, Weill Cornell Medicine, New York, NY, E-mails: mar9227@med.cornell.edu, myl2003@med.cornell.edu, and kad9040@med.comell.edu. Paul L. A. M. Corstjens, Department of Cell and Chemical Biology, Leiden University Medical Center, Leiden, The Netherlands, E-mail: p.corstjens@lumc.nl. Govert J. van Dam, Department of Parasitology, Leiden University Medical Center, Leiden, The Netherlands, E-mail: g.j.van_dam@lumc.nl. Humphrey D. Mazigo, Department of Parasitology, Catholic University of Health and Allied Sciences, Mwanza, 
Tanzania, E-mail: humphreymazigo@gmail.com. Jennifer A. Downs, Department of Medicine, Weill Cornell Medicine, New York, NY, and Department of Medicine, Bugando Medical Centre, Mwanza, Tanzania, E-mail: jna2002@med.cornell.edu.

\section{REFERENCES}

1. Kaonga P, Kaimoyo E, Besa E, Zyambo K, Sinkala E, Kelly P, 2017. Direct biomarkers of microbial translocation correlate with immune activation in adult Zambians with environmental enteropathy and hepatosplenic schistosomiasis. Am J Trop Med Hyg 97: 1603-1610.

2. Munford RS, 2016. Endotoxemia-menace, marker, or mistake? $J$ Leukoc Biol 100: 687-698.

3. Onguru D, Liang Y, Griffith Q, Nikolajczyk B, Mwinzi P, GanleyLeal L, 2011. Human schistosomiasis is associated with endotoxemia and toll-like receptor 2- and 4-bearing B cells. Am J Trop Med Hyg 84: 321-324.

4. Gnauck A, Lentle RG, Kruger MC, 2016. Chasing a ghost? Issues with the determination of circulating levels of endotoxin in human blood. Crit Rev Clin Lab Sci 53: 197-215.

5. Hurley JC, Guidet B, Offenstadt G, Maury E, 2012. Endotoxemia and mortality prediction in ICU and other settings: underlying risk and co-detection of gram negative bacteremia are confounders. Crit Care 16: R148.

6. Corstjens PL et al., 2015. Tools for diagnosis, monitoring and screening of Schistosoma infections utilizing lateral-flow based assays and upconverting phosphor labels. Parasitology 141: 1841-1855.

7. Berhe $\mathrm{N}$ et al., 2004. Variations in helminth faecal egg counts in Kato-Katz thick smears and their implications in assessing infection status with Schistosoma mansoni. Acta Trop 92: 205-212.

8. Associates of Cape Cod, 2017. Limulus Amebocyte Lysate PYROTELL-T. Packag Insert (PN000845).

9. Reich J, Lang P, Grallert H, Motschmann H, 2016. Masking of endotoxin in surfactant samples: effects on Limulus-based detection systems. Biologicals 44: 417-422.
10. Laugrette F, Pineau G, Vors C, Michalski M, 2015. Endotoxemia analysis by the Limulus amoebocyte lysate assay in different mammal species used in metabolic studies. J Anal Bioanal Tech 6: 251.

11. Dawson $M, 2005$. Interference with LAL test and how to address it. LAL Updat 22: 1-5.

12. Roth R, Levin F, Levin J, 1990. Optimization of detection of bacterial endotoxin in plasma with the Limulus test. J Lab Clin Med 116: 153-161.

13. Wong J, Davies N, Jeraj H, Vilar E, Viljoen A, Farrington K, 2016. A comparative study of blood endotoxin detection in haemodialysis patients. J Inflamm (Lond) 13: 24.

14. Gnauck A, Lentle RG, Kruger MC, 2015. The Limulus amebocyte lysate assay may be unsuitable for detecting endotoxin in blood of healthy female subjects. J Immunol Methods 416: 146-156.

15. Welsh FKS, Farmery SM, MacLennan K, Sheridan MB, Barclay GR, Guillou PJ, Reynolds JV, 1998. Gut barrier function in malnourished patients. Gut 42: 396-401.

16. Rogawski McQuade ET et al., 2019. Seasonal food insecurity in Haydom, Tanzania, is associated with low birthweight and acute malnutrition: results from the MAL-ED study. Am J Trop Med Hyg 100: 681-687.

17. Ssempiira J, Kissa J, Nambuusi B, Mukooyo E, Opigo J, Makumbi F, Kasasa S, Vounatsou PS, 2018. Interactions between climatic changes and intervention effects on malaria spatiotemporal dynamics in Uganda. Parasite Epidemiol Control 3: e00070.

18. Rumisha SF, Smith T, Abdulla S, Masanja H, Vounatsou P, 2013. Assessing seasonal variations and age patterns in mortality during the first year of life in Tanzania. Acta Trop 126: 28-36.

19. Leopold SJ et al., 2019. Identifying the components of acidosis in patients with severe Plasmodium falciparum malaria using metabolomics. J Infect Dis 219: 1766-1776.

20. Ahmed T, Azam MA, Armed N, Jamil KM, Hassan F, Ogura N, Tamura H, Yokochi T, 2004. Detection of endotoxin in sera from children hospitalized for treatment of diarrhea in Bangladesh. J Endotoxin Res 10: 223-228. 